\title{
Electrical Power Quality Improvement through Modeling and Optimization of Passive Harmonic Filter
}

\author{
Sina A. Sahidaini \\ Department of Electrical Engineering, College of Engineering \\ Mindanao State University-IIT \\ Iligan City, Philippines \\ College of Maritime Education \\ Zamboanga State College of Marine Sciences and Technology \\ Zamboanga City, Philippines
}

\begin{abstract}
This paper studies on the harmonic distortion and the methods to mitigate the harmonics for a system with Load variation and different harmonic distortion level. A group of passive shunt filter are designed to reduce harmonic distortion and correct power factor. The proposed method optimize the group filter configuration which is suitable for load and distortion level variation to get best scenario out of passive harmonic filters. Maximum level for power factor as long as it is positive, consider as constraint and minimizing harmonics distortion index such as Total Harmonic Distortion (THD) and its Individual Harmonic Distortion (IHD) content are factors for objective function. Genetic Algorithm has been used as Optimization technique and software to simulate are MATLAB for genetic algorithm and ETAP for filter simulation in power system.
\end{abstract}

Keywords--Load Variation, Harmonic Distortion, Power Quality.

\section{INTRODUCTION}

Recently, there is an increasing concern about the environment. The need to generate pollution-free energy has triggered considerable effort toward renewable energy (RE). RE sources such as solar, wind, flowing water and biomass offer the promise of clean and abundant energy. They do not generate any greenhouse gases and are inexhaustible. Solar Power, in particular, is especially attractive for Philippines. In the era of renewable energy, wind power and solar Energy are mostly developed and matured technology around the globe. It is converted into a more convenient alternating current (AC) power through an inverter system. [1]

Advance in technology of semiconductor devices has led to a revolution in electronic technology over the past decade and have more tendency in future.

However rise in the Power Quality problem is due to power equipment which include adjustable-speed motor drives (ASDs), electronic power supplies, direct current (DC) motor drives, battery chargers, electronic ballasts. Power sources act as non-linear loads, drawing a distorted waveform that contains harmonics. These harmonics can cause problems ranging from telephone transmission interference to degradation of conductors and insulating material in motors and transformers. Therefore it is important to gauge the total effect of these harmonics [2]. Schwanz and Bollen have conducted a comprehensive review on solutions of harmonic mitigation techniques using active and passive filters [3]. Jain and Sing estimated harmonics in modern power systems narrowing down key issues and challenges [4].

A paper presented by Adel M. Sharaff, Michael E. Fisher a novel optimization design technique for power system shunt filters to ensure harmonic reduction and noise mitigation on the electrical utility grid. The proposed filter design is based on the minimization of a composite objective function depicting the three main filter objectives of minimum harmonic current penetration into the electric grid system, maximum current absorption by the harmonic filter and minimum harmonic voltage distortion at points of common coupling.

Theory for the Design of C-type Filter by Yao XIAO, Jie ZHAO, Shijie MAO Based on the theoretical analysis, the paper introduces an algorithm for calculating the parameters of C-type filter that has not been published before. Firstly, the relationships of all parameters are established and discussed. Then, Parameters with least cost of the filter are also derived. Finally, the method is testified by the parameters of C-type filters having been used in some HVDC projects. Young-Sik Cho and Hanju Cha present how to select tuning factor and quality factor in designing of a single-tuned passive harmonic filter. Tuning factor and quality factor must be considered before a decision of filter parameters(R, L and C). Rampant rise in population, energy demand, economic growth and emissions have popularized renewable energy sources [6-7]. Flow of currents in longer HVDC and HVAC lines and overloaded distribution transformers cause line losses [8]

\section{FILTER DESIGN}

The most common type of passive filter is the singletuned "notch" filter. This is the most economical type and is frequently sufficient for the application. The notch filter is series-tuned to present a low impedance to a particular harmonic current and is connected in shunt with the power system. Notch filters can provide power factor correction in addition to harmonic suppression. In fact, power factor correction capacitors may be used to make notch filters.

The notch harmonic $\mathrm{h}$ notch is related to the fundamental frequency reactance by:

$h_{\text {notch }}=\sqrt{\frac{X_{e}}{3 X_{F}}}$ 
Note that XC in this case is the reactance of one leg of the delta rather than the equivalent line-to-neutral capacitive reactance. If phase-to phase voltage and three-phase kvar are used to compute $\mathrm{XC}$, as previously described, the factor 3 would be omitted. Also, filters must be designed with the capacity of the bus in mind. The temptation is to size the current-carrying capability based solely on the load that is producing the harmonic. However, a small amount of background voltage distortion on a very strong bus may impose excessive duty on the filter.

(a) Typical low-voltage filter configuration

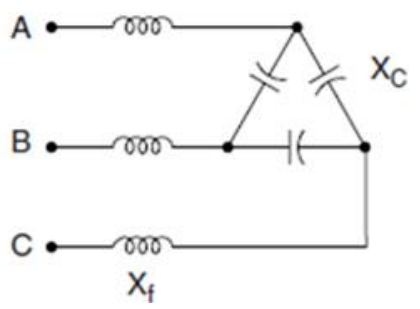

(b) Equivalent circuit of system with filter
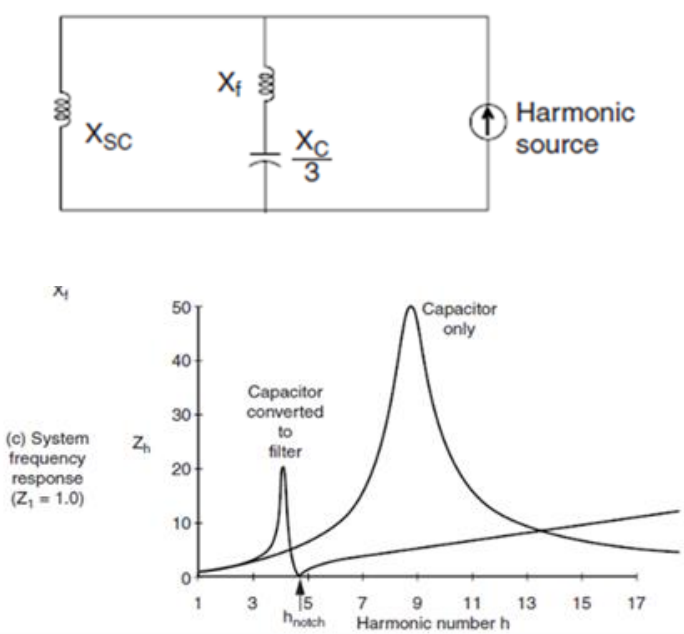

Fig. 1 Creating a fifth-harmonic notch filter and its effect on system response.

\section{A. Harmonic Filter Design Procedure}

In order to design a harmonic filter, information about the local power system, including environmental data, is required. Power system information includes characteristics, such as:

The nominal line-to-line voltage,

- $\quad$ Typical equipment Basic Insulation Level (BIL) for the system voltage level,

- $\quad$ Fundamental frequency,

- System configuration, and

- Impedance of system components

A clear understanding of equipment location (i.e. indoor or outdoor), operating constraints, equipment current duty-cycle, switching operation rates, environmental data (such as ambient temperature and wind loading), harmonic measurements or manufacturer harmonic characteristics, is important to consider before starting the filter design process.

\section{B. Determine Harmonic Filter Bank kVAr Size}

As described previously, the filter equipment provides the system with capacitive, reactive power for power factor improvement, which helps maintain voltage during heavy loads, in addition to filtering the harmonics. The "Effective kVAr" size (2) of the filter depends on the capacitive, reactive power requirements for power factor, as well as voltage control, where the required value is commonly determined by power flow programs.

$Q_{\text {eff }}[$ Mvar $]=\frac{K V_{L-L \text { sys }}^{2}}{X_{\text {eff }}}$

\section{Select Initial Harmonic Filter Tuning}

Filter tuning is necessary to reduce harmonic voltage and current distortion, in order to meet the required harmonic performance. The harmonic filter will typically be tuned to the lower frequency of the most significant harmonic. However, it is recommended that the harmonic filter be tuned between 3 to $15 \%$ below the desired frequency to provide sufficient harmonic filtering, and also to allow room for possible filter detuning. Harmonic filter performance across the whole frequency spectrum, at the filter location side (for both normal and contingency conditions) should be considered.

If the level of harmonic distortion is not crucial, the customer may want to avoid overloading the harmonic filter capacitor with harmonic currents, without creating resonance with the system. Then the filter has to be ungrounded to avoid 3rd harmonic resonance with system and tuned below the 5th harmonic to avoid resonance at the characteristic harmonic.

\section{Optimize the Harmonic Filter Configuration to Meet Harmonic Guidelines}

The IEEE Standard 519-1992 provides recommendations to satisfy harmonic distortion limits. The filter is supposed to meet current and voltage distortion requirements for normal system configuration, as well as abnormal conditions. In cases where the distortion levels remain high, a new parallel resonance with the system may be a possible cause. Filter retuning or multiple-tuned harmonic filter installations can help to solve this problem:

1. The number of harmonic filters.

2. Filter tuning and side locations.

3. Values of capacitance, inductance and reactance,

4. Allowable tolerances for capacitance, inductance and resistance, 
5. $\mathrm{Q}(\mathrm{X} / \mathrm{R}$ ratio) of the harmonic filter reactor at the tuned frequency,

6. As well as steady-state energy dissipation requirements for the harmonic filter resistors,

Can be the results of the harmonic study analysis. The harmonic voltage and current spectra, with fundamental and all significant harmonic frequencies for normal and contingency system conditions, is also important outcome of the analysis.

There is a common practice to use a harmonic simulation programs for possible operating conditions over frequency spectrum of the harmonic loads, meanwhile for a simple system this can be manually estimated.

\section{E. Series (Single-Tuned) Filter}

Figure 2 shows the circuit schematic and a typical impedance characteristic for the series or single-tuned filter.

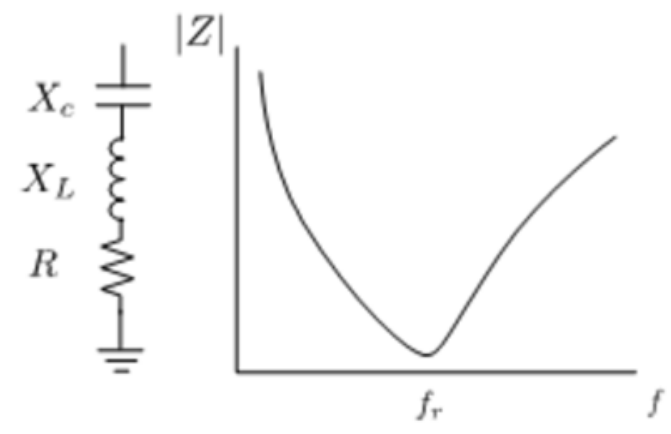

Fig 2. Series (single-tune) Filter

This filter is tuned to suppress a single frequency and is designed based on three quantities:

1. The harmonic current order that requires blocking,

2. The capacitive reactive power that it is going to provide,

3. And its quality factor.

4. The voltage level and the fundamental frequency, which are given by the

System, must also be considered during the design process. In summary, the values used to define the input parameters are:

$\mathrm{h} \quad$ Tuning point of the filter (harmonic order)

$Q_{c}$ Reactive power of the filter [MVAr]

Q Quality factor

F System frequency $[\mathrm{Hz}]$

V

System voltage $[\mathrm{kV}]$
The quality factor is a quantity that defines the bandwidth of the filter and, in this case, is expressed as the ratio between the reactance and the resistance of the filter. A typical range for $\mathrm{Q}$ is between 30 and 60 .

The following equations can be applied for designing the filter:

$$
\begin{aligned}
& C=\frac{Q_{r}}{2 \pi f v^{2}} \\
& X=\frac{1}{2 \pi f h C}=\sqrt{\frac{L}{C}} \\
& L=\frac{X}{2 h f} \\
& Q=\frac{2 \pi f L}{R} \\
& R=\frac{1}{2 \pi f C}
\end{aligned}
$$

Where, $\mathrm{X}$ is the reactance of the inductor or the capacitor at the tuned frequency. [5]

\section{F. GENETIC ALGORITHM}

Genetic algorithm is one of the main paradigms within evolutionary algorithms, which uses an analogy of nature. GA are a part of evolutionary computing, which is a rapidly growing area of artificial intelligence. These are search algorithms based on the mechanics of natural selection and natural genetics. It is an example of iterative search procedure that uses random choice as a tool to guide a highly explorative search through a coding of a parameter space and the reliability is improved by the principle of natural genetics. GA mimic the evolutionary process in nature, which is based on Darwin's theory called "the survival of the fittest", only the fittest individuals likely to survive and reproduce. In GA, instead of trying to optimize a single solution, works with a population of candidate solutions that are encoded as chromosomes. These chromosomes are having separate genes that represent the independent variables for the problem at hand. Algorithm begins with a set of solutions called population. New combinations of genes are generated from previous ones by exchanging segments of genetic material among chromosomes (known as "crossover"). In every generation, a new set of artificial creatures (strings) are created using bits and pieces of the fittest of the old and an occasional new part is tried for good measure. This is motivated by a hope, that the new population will be better than the older one. A fitness function must be provided for evaluating each string. Each solution is associated with a fitness value, based on the fitness function, to reflect how good it is. Solutions which are selected to form new solutions (offspring) are reproduced according to their fitness. 


\section{PROBLEM FORMULATION}

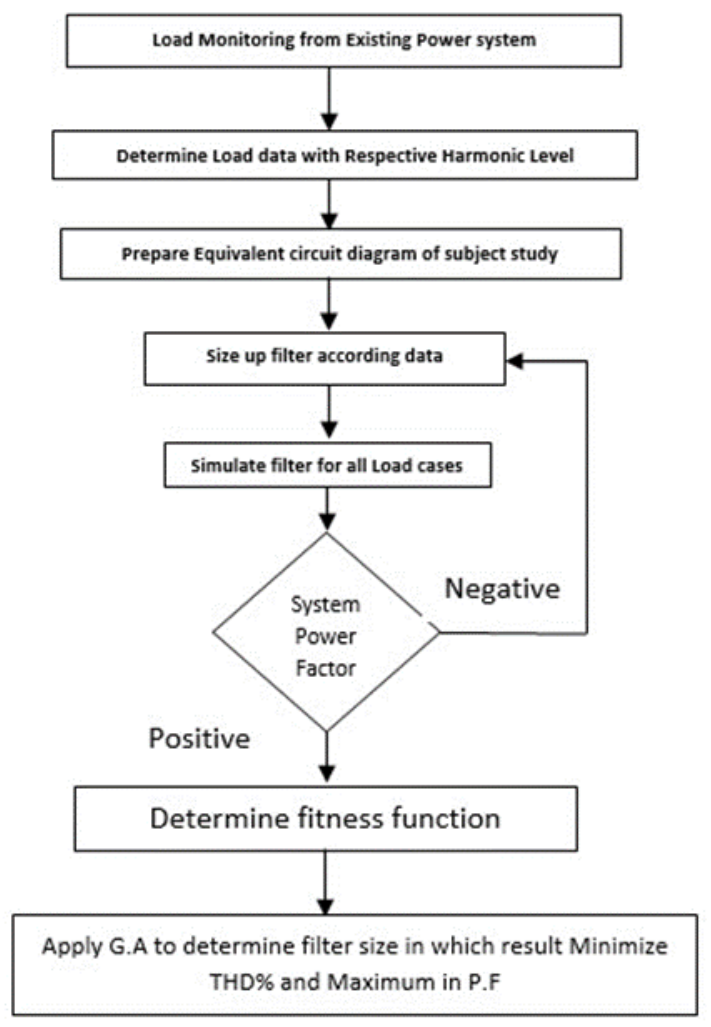

Fig. 3. Flowchart for Harmonic Mitigation

Based on data were collected and statistical information, following cases are selected to be simulated:

TABLE 1. INHD $\%$ for $3^{\text {rd }}$ and $5^{\text {th }}$

\begin{tabular}{|l|c|c|c|c|c|c|}
\hline & \multicolumn{2}{|c|}{ Line A } & \multicolumn{2}{c|}{ Line B } & \multicolumn{2}{c|}{ Line C } \\
\cline { 2 - 7 } & $3^{\text {rd }}$ & $5^{\text {th }}$ & $3^{\text {rd }}$ & $5^{\text {th }}$ & $3^{\text {rd }}$ & $5^{\text {th }}$ \\
\hline Case A & 10 & 14 & 10 & 15 & 12 & 15 \\
\hline Case B & 14 & 15 & 15 & 16 & 16 & 17 \\
\hline Case C & 16 & 16 & 16 & 17 & 20 & 20 \\
\hline Case D & 17 & 21 & 19 & 23 & 25 & 28 \\
\hline
\end{tabular}

TABLE 2. System Power

\begin{tabular}{|c|c|c|c|}
\hline & KW & Kvar & P.F \\
\hline Case A & 52.52 & 26.37 & 89.4 \\
\hline Case B & 61.2 & 36.31 & 86.0 \\
\hline Case C & 64.66 & 45.79 & 81.6 \\
\hline Case D & 69.26 & 54.32 & 78.6 \\
\hline
\end{tabular}

TABLE 3-system Harmonic Before implementation of Harmonic Filter

\begin{tabular}{|l|c|c|c|c|c|}
\hline & THD\% & I(\%)5th & I(A) 5th & I(\%)7th & I(A) 7th \\
\hline Case A & 16.66 & 14.573 & 20.4 & 7.331 & 10.3 \\
\hline Case B & 18.71 & 15.924 & 27 & 7.93 & 13.4 \\
\hline Case C & 21.28 & 16.512 & 31.2 & 9.192 & 17.3 \\
\hline Case D & 28.36 & 24.08 & 50.4 & 9.592 & 20.1 \\
\hline
\end{tabular}

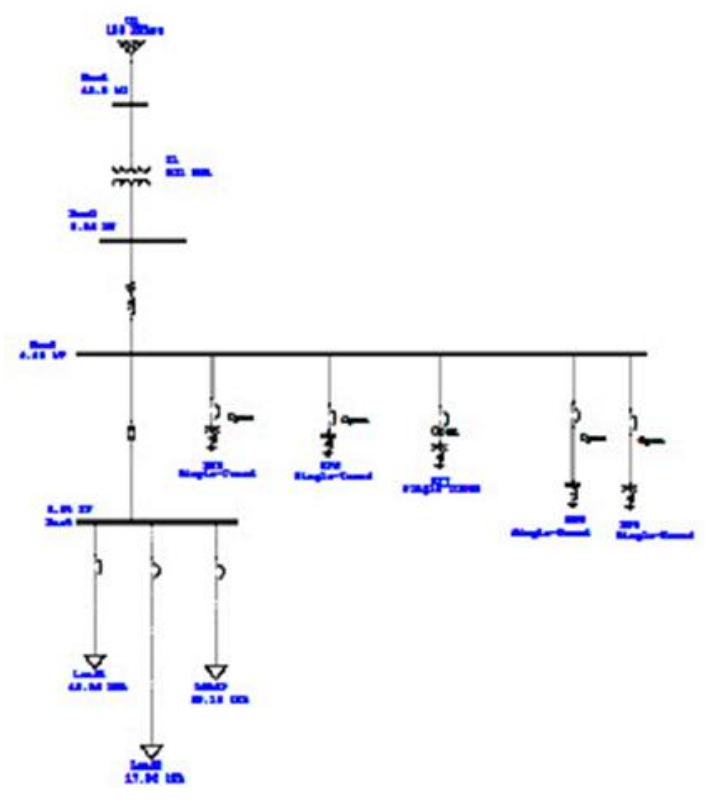

Fig. 4. Equivalent circuit Diagram

Five passive, three phase filter as shown in figure 4. Are used for 5 th and 7 th harmonic mitigation. While load are connected are in delta, with 240 line voltage at low side and $13.6 \mathrm{kv}$ in high side and primary of transformer. $\mathrm{Y} / \Delta$ connection of transformer minimize the 3rd harmonic at PCC.

\section{RESULT AND DISCUSSION}

Case A

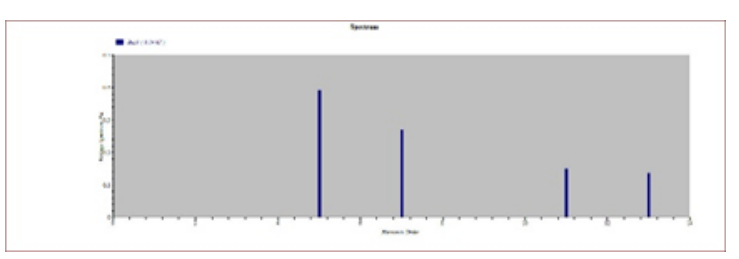

Fig. 5. Harmonic spectrum for Case A without filter.

Case a consider minimum average load which can consider for actual operation of power system therefor computation for Filter for case A are presented in table 4.

Table 4. Computed Filter size for eliminating $5^{\text {th }}$ harmonic only for simulation

\begin{tabular}{|c|c|c|c|c|c|}
\hline Qeff(Kvar) & $\boldsymbol{Q}_{\boldsymbol{C}}(\mathrm{Kvar})$ & $\boldsymbol{X}_{\boldsymbol{L}}(\Omega)$ & $\boldsymbol{V}_{\boldsymbol{G}}$ & $\mathrm{C}(\mu \mathrm{F})$ & $\mathrm{L}(\mathrm{mH})$ \\
\hline 10.4 & 3.614 & 0.6910 & 250 & 153.26 & 1.836 \\
\hline 10.6 & 3.6770 & 0.6799 & 250 & 156.21 & 1.8017 \\
\hline 10.8 & 3.7500 & 0.6667 & 250 & 159.15 & 1.768 \\
\hline 11.00 & 3.8200 & 0.6545 & 250 & 162.1 & 1.736 \\
\hline 11.20 & 3.8800 & 0.6428 & 250 & 165.05 & 1.7052 \\
\hline 11.40 & 3.9580 & 0.6316 & 250 & 168 & 1.6753 \\
\hline 11.60 & 4.0280 & 0.6207 & 250 & 170.95 & 1.6464 \\
\hline 11.80 & 4.0970 & 0.6102 & 250 & 173.88 & 1.618 \\
\hline 12.00 & 4.1667 & 0.6000 & 250 & 176.84 & 1.5915 \\
\hline 12.20 & 4.2360 & 0.5901 & 250 & 179.79 & 1.5654 \\
\hline 12.40 & 4.3050 & 0.5806 & 250 & 182.73 & 1.5402 \\
\hline 12.60 & 4.3750 & 0.5714 & 250 & 185.68 & 1.5158 \\
\hline 12.80 & 4.4440 & 0.5625 & 250 & 188.63 & 1.492 \\
\hline 13.00 & 4.5140 & 0.5539 & 250 & 191.56 & 1.469 \\
\hline 13.180 & 4.5780 & 0.5461 & 250 & 194.3 & 1.448 \\
\hline
\end{tabular}


Where:

\section{$Q_{\text {eff }}\left(K_{\text {var })}\right.$ : Effective Kvar of filter per Phase \\ $Q_{c}($ Kvar $)$ : Capacitor Kvar per phase}

\section{$X_{L}(\Omega)$ : Filter Inductive resistance of filter \\ $V_{c}$ : Capacitor Voltage \\ $\mathrm{C}(\mu \mathrm{F})$ : Capacitor Size in micro Farad \\ $\mathrm{L}(\mathrm{mH})$ : Inductance of Filter Reactor in mili-Henry}

As far as Power factor is constraint, to kept positive and maximum for case a, two identical $13.18 \mathrm{KV}$ ar are in parallel taking down fifth harmonic for minimum average load, a range from 10.4 Kvar to 13.18 kvar are computed, to be simulated one by one to produce our fitness function for genetic algorithm(GA). The result in table 4 are simulated in three identical filter for case $b$, four identical filter for case $c$ and five identical filter for case d. to ensure that whether case a can be basis for filter configuration, the result must pass constraint of maximum power factor and positive for safer operation and avoid over voltage at Point of Common Coupling PCC.

Table 5. Filter simulation result for case a.

\begin{tabular}{|l|r|r|l|r|r|}
\hline \multicolumn{2}{|l|}{$\boldsymbol{Q}_{\text {eff }}(\boldsymbol{K}$ vadyD $\%$} & $\boldsymbol{I}_{5 \text { th }}$ \% & $\boldsymbol{P}_{\boldsymbol{W}} \boldsymbol{f}$ & \multicolumn{1}{l|}{ P.F } & $\boldsymbol{I}_{5 \text { th }}(\mathrm{A})$ \\
\hline 10.4 & 8.65 & 2.48 & 0.014 & 99.4 & 3.1 \\
\hline 10.6 & 8.58 & 2.248 & 0.014 & 99.48 & 2.8 \\
\hline 10.8 & 8.56 & 2.181 & 0.014 & 99.55 & 2.8 \\
\hline 11.00 & 8.54 & 2.144 & 0.015 & 99.62 & 2.7 \\
\hline 11.20 & 8.52 & 2.105 & 0.015 & 99.69 & 2.7 \\
\hline 11.40 & 8.51 & 2.065 & 0.015 & 99.74 & 2.6 \\
\hline 11.60 & 8.49 & 2.031 & 0.016 & 99.79 & 2.6 \\
\hline 11.80 & 8.48 & 1.999 & 0.016 & 99.84 & 2.5 \\
\hline 12.00 & 8.46 & 1.968 & 0.016 & 99.88 & 2.5 \\
\hline 12.20 & 8.44 & 1.938 & 0.016 & 99.91 & 2.4 \\
\hline 12.40 & 8.45 & 2.006 & 0.017 & 99.94 & 2.5 \\
\hline 12.60 & 8.41 & 1.883 & 0.017 & 99.97 & 2.4 \\
\hline 12.80 & 8.41 & 1.915 & 0.017 & 99.98 & 2.4 \\
\hline 13.00 & 8.39 & 1.882 & 0.017 & 99.99 & 2.4 \\
\hline 13.180 & 8.36 & 0.041 & 0.018 & 100 & 1.803 \\
\hline
\end{tabular}

Simulation result by ETAP are presented in Table 5. In which shows that THD\% is minimum at $13.18 \mathrm{Kvar}$, filter size while result in a power factor unity as well. Taking note that table 5. Are points in which will help us to build up a fitness function for genetic algorithm by interpolation and curve fitting method. So far this is the best filter size but only when the system are on average minimum system load. However this filter range must be simulated for other cases to fit the constraint.

Table 6. Filter simulation result for case b. but base on case a.

\begin{tabular}{|l|l|l|l|l|l|}
\hline$Q_{\text {eff }}\left(K_{\text {I }}\right.$ & FFAD & $I_{\text {5th }} \%$ & $P_{W} f$ & $P . F$ & $I_{\text {5th }}(A)$ \\
\hline 10.4 & 10.39 & 1.891 & 0.014 & 99.61 & 2.8 \\
\hline 10.6 & 10.35 & 1.71 & 0.015 & 99.69 & 2.5 \\
\hline 10.8 & 10.34 & 1.685 & 0.015 & 99.76 & 2.5 \\
\hline 11.00 & 10.32 & 1.655 & 0.015 & 99.83 & 2.4 \\
\hline 11.20 & 10.32 & 1.782 & 0.015 & 99.87 & 2.6 \\
\hline 11.40 & 10.28 & 1.597 & 0.016 & 99.92 & 2.3 \\
\hline 11.60 & 10.26 & 1.57 & 0.016 & 99.96 & 2.3 \\
\hline 11.80 & 10.24 & 1.546 & 0.016 & 99.98 & 2.3 \\
\hline 12.00 & 10.21 & 1.522 & 0.017 & 100 & 2.2 \\
\hline
\end{tabular}

In table 6. The filter of case a. are simulated on case b, but it only fit up to $12 \mathrm{kvar}$, because more than $12 \mathrm{kvar}$, the system power factor become negative. Result that 13.18 kvar filter are not fit for case b. like the processes in case b, must be applied for other cases to find a range which is suitable for all cases. Because compare to active filter passive filters are fixed even though load changes.

TABLE 7. Filter simulation result for case c. but base on case a

\begin{tabular}{|l|l|l|l|l|l|}
\hline $\boldsymbol{Q}_{\text {eff }}$ (Kvat & JHD\% & $\boldsymbol{I}_{5 \text { th }} \%$ & $\boldsymbol{P}_{\boldsymbol{W}} \boldsymbol{f}$ & P.F & $\boldsymbol{I}_{5 \text { th }}(\mathrm{A})$ \\
\hline 10.4 & 14.51 & 1.554 & 0.014 & 99.75 & 2.4 \\
\hline 10.6 & 14.48 & 1.419 & 0.015 & 99.83 & 2.2 \\
\hline 10.8 & 14.45 & 1.394 & 0.015 & 99.9 & 2.2 \\
\hline 11.00 & 14.42 & 1.37 & 0.015 & 99.95 & 2.1 \\
\hline 11.20 & 14.4 & 1.463 & 0.015 & 99.97 & 2.3 \\
\hline 11.40 & 14.36 & 1.322 & 0.016 & 100 & 2 \\
\hline
\end{tabular}

TABLE 8. Filter simulation result for case $d$. but base on case a.

\begin{tabular}{|c|c|c|c|c|c|}
\hline$Q_{\text {eff }}($ Kvar $)$ & THD\% & $I_{5 t h} \%$ & $P_{W} f$ & P.F & $I_{\text {5th }}(\mathrm{A})$ \\
\hline 10.4 & 14.51 & 1.554 & 0.014 & 99.75 & 2.4 \\
\hline 10.6 & 14.48 & 1.419 & 0.015 & 99.83 & 2.2 \\
\hline 10.8 & 14.45 & 1.394 & 0.015 & 99.9 & 2.2 \\
\hline 11.00 & 14.42 & 1.37 & 0.015 & 99.95 & 2.1 \\
\hline 11.20 & 14.4 & 1.463 & 0.015 & 99.97 & 2.3 \\
\hline 11.40 & 14.36 & 1.322 & 0.016 & 100 & 2 \\
\hline
\end{tabular}

Table 5 to 6 are evidently showing sizing filter base on minimum average load result in negative power factor for other cases at case (a) optimal filter size. This process are through for all cases to find a fitness function which is fit for only constraint for all cases. The finding are presented in table 9.

TABLE 9. Acceptable filter size for all cases base on constraint of $\mathrm{pf} \leq 100$.

\begin{tabular}{|l|l|l|l|l|l|}
\hline Qeff(Kvar) & $Q_{C}($ Kvar $)$ & $X_{L}(\Omega)$ & $V_{C}$ & $\mathrm{C}(\mu \mathrm{F})$ & $\mathrm{L}(\mathrm{mH})$ \\
\hline 8 & 2.777 & 0.9 & 250 & 117.89 & 2.387 \\
\hline 8.2 & 2.8472 & 0.878 & 250 & 120.84 & 2.3291 \\
\hline 8.4 & 2.9166 & 0.8571 & 250 & 123.79 & 2.274 \\
\hline 8.6 & 2.986 & 0.8372 & 250 & 126.73 & 2.221 \\
\hline 8.8 & 3.0555 & 0.8182 & 250 & 129.68 & 2.17 \\
\hline 9 & 3.125 & 0.8 & 250 & 132.63 & 2.1221 \\
\hline 9.2 & 3.194 & 0.7826 & 250 & 135.57 & 2.0759 \\
\hline 9.4 & 3.263 & 0.766 & 250 & 138.52 & 2.0318 \\
\hline 9.6 & 3.333 & 0.75 & 250 & 141.47 & 1.9894 \\
\hline 9.8 & 3.403 & 0.7347 & 250 & 144.42 & 1.9488 \\
\hline 10 & 3.472 & 0.72 & 250 & 147.37 & 1.9098 \\
\hline 10.2 & 3.542 & 0.7059 & 250 & 150.31 & 1.872 \\
\hline 10.4 & 3.6111 & 0.6923 & 250 & 153.26 & 1.8364 \\
\hline 10.6 & 3.6806 & 0.6792 & 250 & 156.21 & 1.802 \\
\hline 10.8 & 3.75 & 0.6667 & 250 & 159.15 & 1.7684 \\
\hline
\end{tabular}

According table 9 are more convenient with the constraint of $\mathrm{pf} \leq 100$. Table 9 are proper to build up the fitness function for Genetic algorithm in the way that each result in simulation is representing a function in which decision variable is the $Q_{\text {eff }}$, only and it is depend on $X_{L}$ of reactance and $Q_{c}$ Kvar of the filter.

Therefore, based on the result on table 9 we formulate our optimization process as follows: 
TABLE 10. Optimization process

\begin{tabular}{|l|l|l|}
\hline & Optimization & Process \\
\hline 1 & Decision Variable & $Q_{\text {eff }}$ filter \\
\hline 2 & Objective function & minimize : \\
& & THD\%, IHD (\%) \\
& & IHD(A) \\
& & Maximize: \\
\hline 3 & Constraint & System Power factor \\
\hline
\end{tabular}

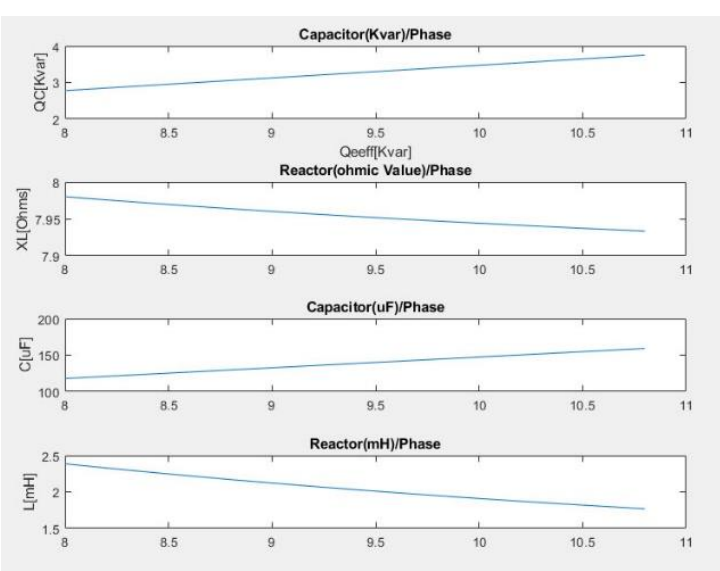

Fig. 6. Curve fitting of Table 9 done by Matlab interpolation

Figure 6 is made out of data in table 9. By Matlabe curve fitting interpolation Informative regarding filter size throughout harmonic mitigation, wherein how filter size change in capacitor and reactance for duration of finding optimize size of filter.

TABLE 11. Result of filter optimization for 5th harmonic only

\begin{tabular}{|l|l|l|l|}
\hline & THD $\%$ & $\mathrm{I}(\%) 5^{\text {th }}$ & P.F \\
\hline Case A & 8.56 & 2.181 & 99.55 \\
\hline Case B & 10.34 & 1.685 & 99.76 \\
\hline Case C & 14.45 & 1.394 & 99.9 \\
\hline Case D & 16.37 & 1.698 & 99.99 \\
\hline
\end{tabular}

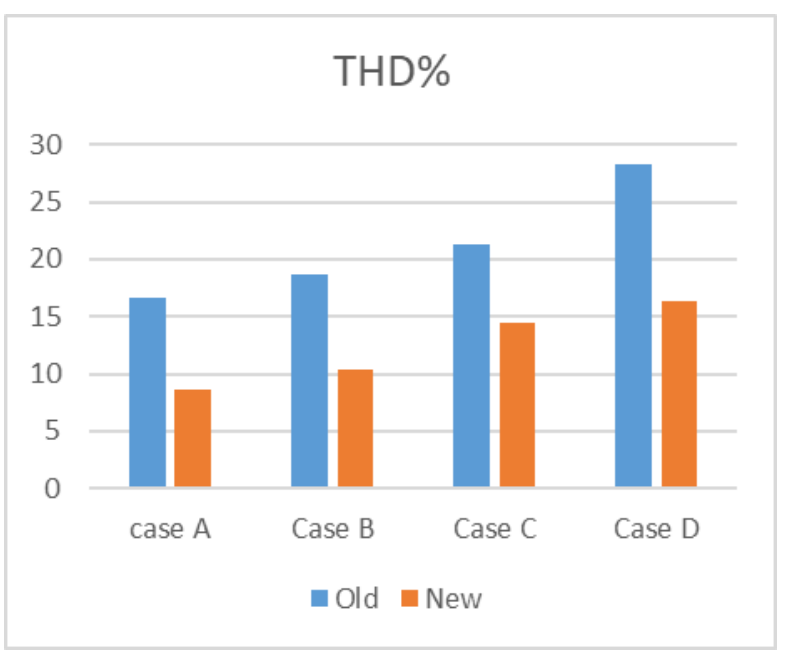

Fig. 7. $5^{\text {th }}$ harmonic filter only result for THD $\%$

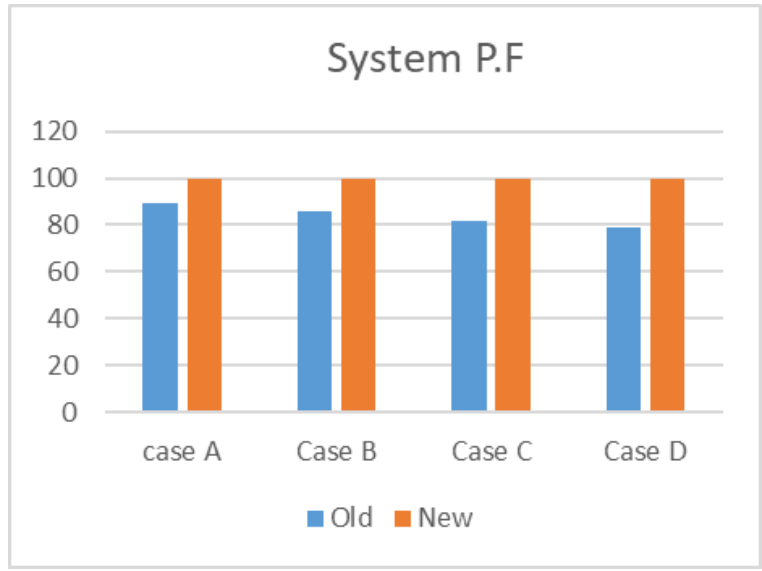

Figure 8. $5^{\text {th }}$ harmonic filter result for IHD\%

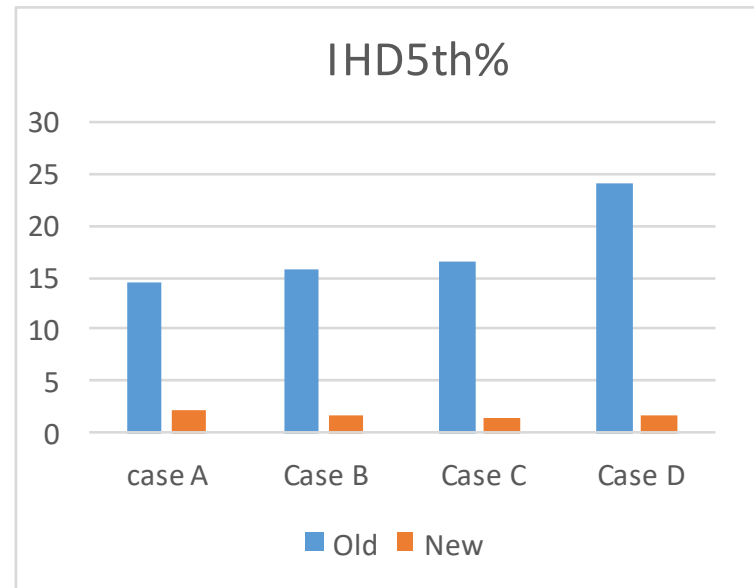

Figure 9. $5^{\text {th }}$ harmonic filter Result for System P.F

TABLE 12. . Result of optimization for 7th harmonic filter only

\begin{tabular}{|c|c|c|c|}
\hline & THD\% & $\mathrm{I}(\%) 7^{\text {th }}$ & P.F \\
\hline Case A & 18.21 & 0.425 & 99.55 \\
\hline Case B & 22.18 & 0.322 & 99.76 \\
\hline Case C & 26.29 & 3.19 & 99.73 \\
\hline Case D & 40.46 & 2.634 & 99.91 \\
\hline
\end{tabular}

THD\%

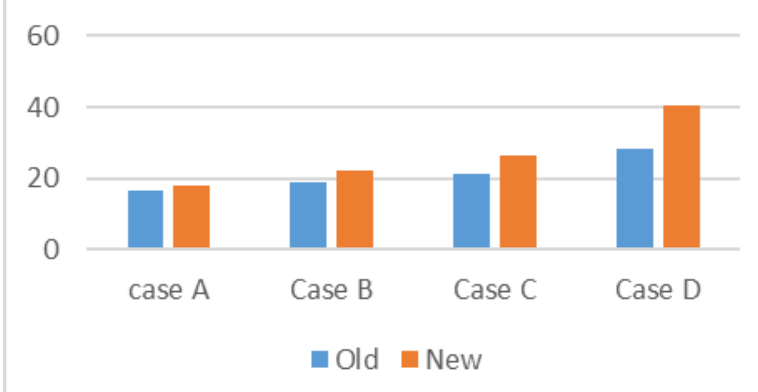

Fig. $9.7^{\text {th }}$ only harmonic filter result for THD\% 


\section{IHD 7th( \% )}

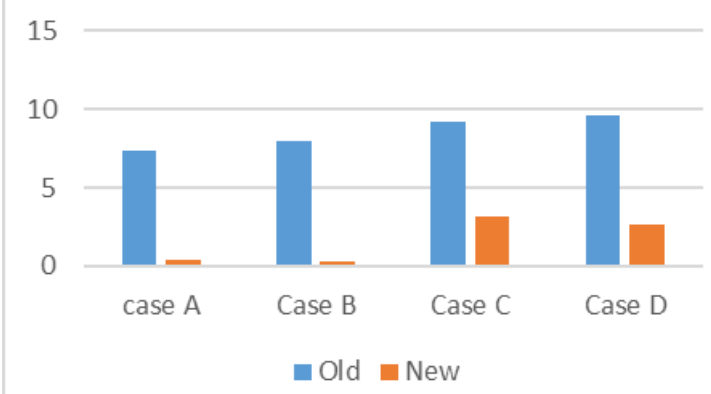

Figure 10. 7th only harmonic filter result for IHD\%

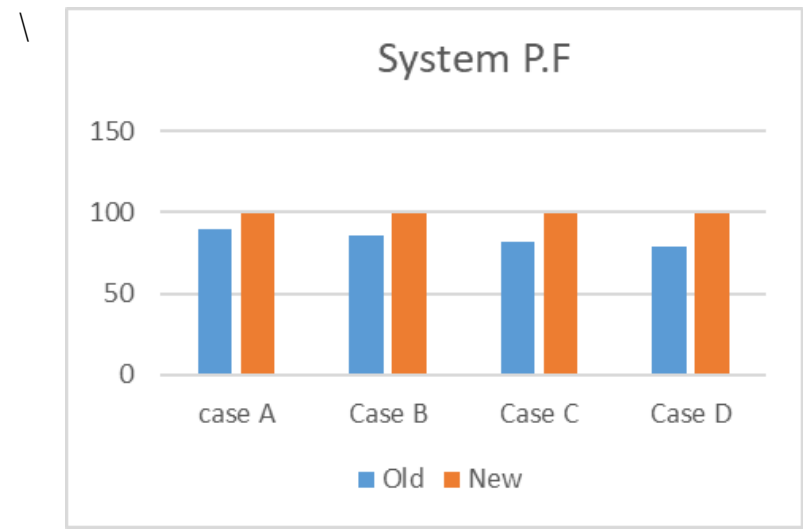

Fig. 11. 7th only harmonic filter result for system P.f

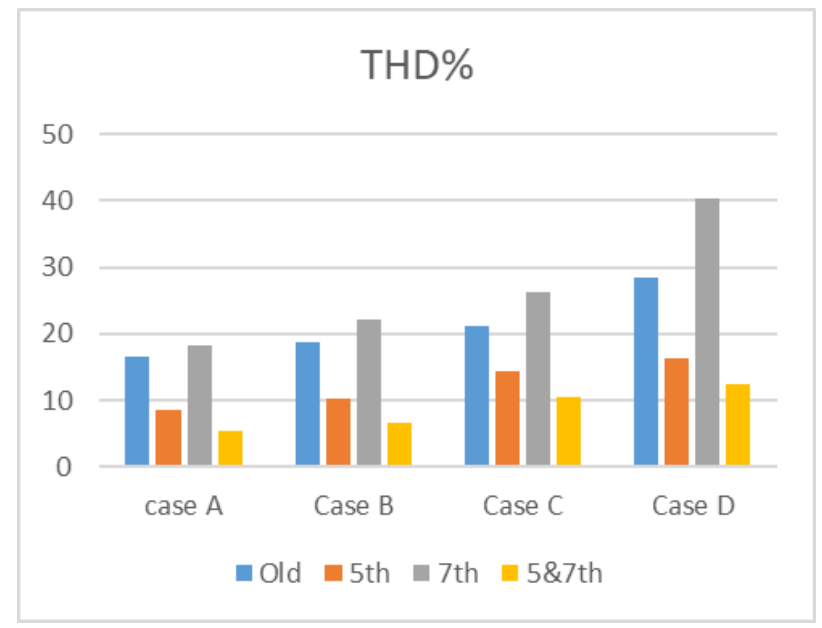

Fig. 12. THD\% for all cases and different filter set

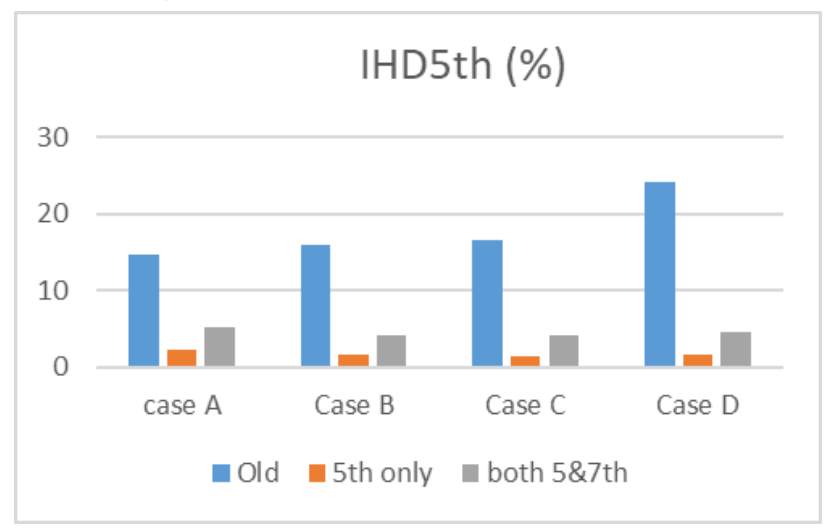

Fig. 13. IHD\% 5th for all cases and different filter set up

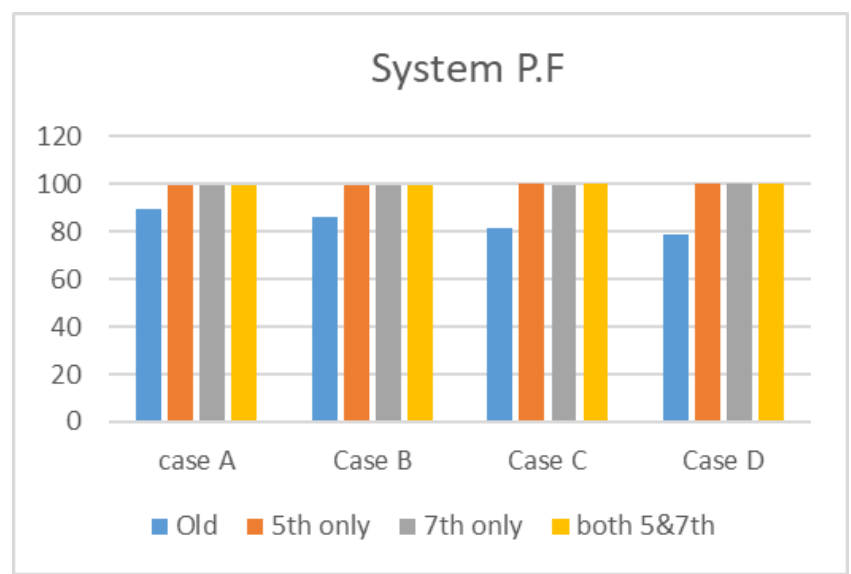

Fig.14. System Power Factor for different Load cases

Four cases for load study and five filters configuration are optional, and depend on system study and monitoring period. In Figure 14. Represent propose filter configuration constraint has been well maintained for all load cases and different filter setup and system power factor well maintained. In $5^{\text {th }}$ only filter configuration has a better limiting on their respective tuning frequency, to limit Individual harmonic. In $7^{\text {th }}$ only harmonic THD\% system for all cases, dramatically has been increased, therefor contradicting objective function to minimize THD\%. In spite minimizing IHD $7^{\text {th }} \%$ in lowest for all cases compare to other filter set up. To achieve optimization, objective function must be fulfilled.

Filters configuration outcomes are summarized in table 14. And assessed by objective function.

\begin{tabular}{|c|c|c|c|}
\hline \multirow{2}{*}{$\begin{array}{c}\text { Filter } \\
\text { configuration }\end{array}$} & \multicolumn{3}{|c|}{ Objective Function } \\
\hline & THD $\%$ & IHD\% & P.F \\
\hline $\begin{array}{l}5^{\mathrm{TH}} \text { harmonic } \\
\text { filter }\end{array}$ & Reduced & Reduced & Optimized \\
\hline $\begin{array}{l}7^{\text {th }} \text { Harmonic } \\
\text { Filter }\end{array}$ & Worsen & Reduced & Optimized \\
\hline $\begin{array}{l}\text { Both } 5^{\text {th }} \& 7^{\text {th }} \\
\text { harmonic filter }\end{array}$ & Optimized & Optimized & Optimized \\
\hline
\end{tabular}

\section{CONCLUSION}

In this paper, study on harmonic mitigation by optimizing passive harmonic filter set for load changing and THD\% variation has been carried out. Optimized filter configuration by implementing Genetic Algorithm on various fitness function or multi-objective function to maximize system power factor, minimize THD\% and IHD\% for 5th and 7th harmonic. To avoid over voltage, power factor must be Less than 1.0, and it is consider optimization constraint. The result shows that 5 th harmonic has acceptable performance and in all cases perform well while 7th harmonic filter worsen THD\%. A combination of 5th and 7 th harmonic filter configuration has better performance compare to other set up. However we have to take note filter set up depend on period of data collection and power system output in data analysis, for example in this study data were collected for a period of one week, if it is extended can have smoother or clear view of load changes in system will be provided. The importance we have to figure it out how load in system are changing and how it is effecting system harmonic. 
The advantage of this study optimization carried out by finding Qeff of filter, therefor it can be implemented to not just single tune but C-type filter as well. The hassle of solving for filter elements is eased by $Q_{\text {eff }}$, which is the same for either single tune or c-type filter.

\section{ACKNOWLEDGMENT}

The authors acknowledge the Mindanao State UniversityIligan Institute of Technology (MSU-IIT), The Zamboanga State College of Marine Sciences and Technology for their support for performing this work. This work was supported and funded by Commission on Higher Education (CHED) under CHED K to 12 scholarship program.

\section{REFERENCES}

[1] G. Eason, B. Noble, and I.N. Sneddon, "On certain integrals of Lipschitz-Hankel type involving products of Bessel functions," Phil. Trans. Roy. Soc. London, vol. A247, pp. 529-551, April 1955. (references)

[2] J. Clerk Maxwell, A Treatise on Electricity and Magnetism, 3rd ed., vol. 2. Oxford: Clarendon, 1892, pp.68-73.

[3] I.S. Jacobs and C.P. Bean, "Fine particles, thin films and exchange anisotropy," in Magnetism, vol. III, G.T. Rado and H. Suhl, Eds. New York: Academic, 1963, pp. 271-350.

[4] K. Elissa, "Title of paper if known," unpublished.

[5] R. Nicole, "Title of paper with only first word capitalized," J. Name Stand. Abbrev., in press.

[6] Y. Yorozu, M. Hirano, K. Oka, and Y. Tagawa, "Electron spectroscopy studies on magneto-optical media and plastic substrate interface," IEEE Transl. J. Magn. Japan, vol. 2, pp. 740-741, August 1987 [Digests 9th Annual Conf. Magnetics Japan, p. 301, 1982].

[7] M. Young, The Technical Writer's Handbook. Mill Valley, CA: University Science, 1989. 